\title{
INFORMATION SYSTEMS CHALLENGES OF HEALTHCARE IN THE UNITED STATES
}

\author{
Gary Janchenko, University of Pittsburgh, gary.janchenko@pitt.edu \\ Anthony Rodi, D.Sc., University of Pittsburgh, afrodi@katz.pitt.edu \\ Michael J. Donohoe, D.Sc. National Defense University, m.j.donohoe.civ@ndu.edu
}

\begin{abstract}
The Healthcare Industry in the United States has traditionally lagged behind other industries in the adoption of Information Systems and Technologies. It has become highly regulated due in part to the introduction of HIPAA (Healthcare Insurance Portability and Accountability Act). As a result of this regulation, Healthcare technology has become highly regulated as ongoing challenges occur with the adoption of new technologies. Government policy dictates technology needs even though advances in technology may offer patients the newest features and ease of use in accessing medical information. Using previous research and literature, this paper will seek to understand and explain the challenges and successes experienced in healthcare through regulation, policy changes and requirements for greater efficiencies.
\end{abstract}

Keywords: Information Systems, Healthcare Information Systems, Big Data, IS, Healthcare IT, Privacy, Data Security

\section{INTRODUCTION}

Healthcare in the United States has traditionally lagged behind other industries in the adoption of Information Systems and Technologies. It has become highly regulated due in part to the introduction of HIPAA (Healthcare Insurance Portability and Accountability Act). As a result of this regulation, Healthcare technology has become highly regulated as ongoing challenges occur with the adoption of new technologies. Government policy dictates technology needs even though advances in technology may offer patients the newest features and ease of use in accessing medical information. The scope and complexity of healthcare regulation has made it incredibly difficult for organizations to adopt new technologies. Compared to other industries, they have been relatively slow to adopt technological innovations as a result (Dash, 2016).

The adoption of the Electronic Medical Records (EMR) systems has ushered in a host of new challenges for an industry that is thoroughly entrenched in a physical, paper world. On top of the huge financial burdens that the electronic systems can impose upon a health system come the new challenges with ethics and system security. Problems that used to be more easily solved with a locked chart room now encompass a team of experts, around the clock monitoring and even more regulation. Getting the most out of the systems requires hours of chart abstraction, constant data cleanup and an endless stream of system updates as vendors struggle to keep up with sometimes unrealistic regulatory expectations. The software landscape is strewn with hundreds of vendors and health systems with proprietary applications and data storage frameworks that have made it very difficult for desperate systems to share data about patients but advances in interoperability are making it easier for doctors to get a clear picture of a patient's overall health by federating data from a variety of sources. Though the transition to an electronic world offers significant challenges, tools such as Population Health and other analytics platforms are maturing and allowing providers to take better care of their patients and more closely monitor the composite health of large groups of patients more efficiently and effectively. 


\section{PURPOSE OF THE STUDY}

The purpose of this study was to examine existing research and to determine the effectiveness of mandated Healthcare technology on physician productivity and on improving patient outcomes. It also examines the information technology challenges and advances related to Big Data, Security and Ethics when implementing Electronic Health Record Systems in the United States.

\section{LITERATURE REVIEW}

\section{Government Regulation}

The Health Information Portability and Accountability Act "HIPAA" was established in 1996 by the U.S Department of Health and Human Services "HHS" to provide a framework for the disclosure and use of health records. (HHS.gov, 2016) The Privacy rule's main goal is to provide rules around the proper protection and security of personal health information while defining the uses when the information may be shared with other entities. The rule applies to any entity that stores or transmits health data and includes: clearinghouses, insurance companies and health care providers to name a few. The rule is designed to cover a wide range of personally identifiable health information and not only covers data related to health treatment but also any payments in the provision of any care. The Privacy Rule also includes a provision to define appropriate ways to de-identify patient data and declares that there are no restrictions on the use or disclosure of any health records that have been properly de-identified.

\section{Meaningful Use}

"Meaningful Use" refers to the use of certified EHR (Electronic Health Records) to improve quality, safety, efficiency and to improve overall Healthcare coordination, (Healthit.gov, 2016). The HITECH Act, (Health Information Technology for Economic and Clinical Health) was part of a stimulus package named American Recovery and Reinvestment Act of 2009 (ARRA) provided financial incentives to physicians to promote the adoption and use of Healthcare Information Technology (HIT) and (EHR) Electronic health Records (Blumenthal, 2009). HITECH, in accordance with HIPAA works to ensure data security and portability of electronic health records and requires healthcare organizations to immediately notify of any compromise in patient healthcare data as well as limits its use of data for commercial purposes (Blumenthal, 2009, Healthit.gov, 2016).

\section{THE INFORMATION SYSTEMS CHALLENGES OF HEALTHCARE}

\section{Interoperability}

The healthcare industry is looking for greater interoperability for patient's EHR (Electronic Health Records) so that they are shared and can talk to each other when moving between health systems, (Ghosh, 2014). In addition to moving patient records between different systems, some systems are looking for ways to converge records within the system itself. No single piece of software can meet the needs of each department within a hospital, for example. To give each department the best software for the type of business we often see patient registration and scheduling done within one system and laboratory and diagnostic services documented in another system while the providers themselves are documenting contact time with the patient in a third, disparate system. Outside of the system, the landscape gets increasingly complicated because of the variety of vendors offering a wide range of software packages to meet specific customer needs without regard to how these systems may ever communicate with one another. To bridge the gap between systems, even more vendors are entering the market offering Electronic Data Interchange (EDI) services to help share information thus further complicating the industry.

While the government has been forthcoming on what types of data must be collected by hospitals and outpatient offices, the standards in which everyone must operate have not been so well defined. Historically, apart from standard ICD and CPT coding systems there had been no real frameworks defined on how data should be collected, 
stores and transmitted which has added to the convoluted matrix of systems. To try to entice vendors and users share their patient information across networks electronically, the US government has added to the Meaningful Use program a requirement that systems must share their patient records in a system called a Health Information Exchange. This time, to help define some standards to facilitate the exchange, the government has defined a set of coding standards such as: ICD-10, SNOMED, RxNorm, LOINC and CVX. Furthermore, the data must be exchanged in a standardized format called a Continuity of Care Document (CCD), (Mostashari, (2012).

Despite the framework being set by the government, the full transition to these standards is going to take some time and is likely going to alter the number of vendors in the market. Those vendors that are capable of acquiring talent and have the financial resources to adjust their software to meet the standards or in some cases complete rewrite certain aspects will emerge the market winners and will dominate. Those whom offer software that is not approved by the Centers for Medicare \& Medicaid Services (CMS) will likely lose business and could be forced to shut down. Is it possible that Meaningful Use is causing unnatural changes in the marketplace? Even when the various vendors update their software to meet the current standards there is still the issue with implementation on the side of the hospitals or private practices. Doctors have long grown tired of the seemingly constant system outages and trainings that occur on a consistent basis due to code updates or complete system upgrades. Many argue that they never had to upgrade their paper charts, never experienced any downtime while documenting on paper records and interoperability was a simple as faxing a chart to the requesting office. These system updates are often delayed as much as possible by systems to lessen disruption by combining multiple updates into one downtime event which only makes the timelines for total compliance even longer. Once all systems are using the same standards and communicating with the same document framework there is still the issue of requesting and delivering the patient data which at this time is often a relatively manual process. Within ideal of interoperability lies the notion that systems will somehow automatically request, process and receive the records though the reality is that there is always a person at a computer processing requests one at a time. This is where the benefit EDI services will ultimately come to fruition and will help to enable the translation of records from one system into the standards and send it along to the final destination.

\section{The Use of Analytics}

Data in healthcare is an ongoing concern, from securing it to making sense of the mountains of data. Based on a survey conducted at HIMSS15, a Health IT Conference in 2015, in Chicago, the result of their research indicated that nearly half of healthcare providers were confused and frustrated concerning Big Data Analytics, with one third not knowing what to do with the data that they have, concerning analytics (Bresnick, 2015, Stoltenberg Consulting, 2015). With the volume of data being generated in Healthcare, there is huge interest in how to leverage this data to cut cost and create improvements in health outcomes. "Because of the size and variety of data, there are challenges to analyzing and efficiently using Healthcare big data," (Tan, et al, 2015). Most traditional Healthcare data has been in the physical forms of x-rays, paper files, etc. New data streams in many different forms are flowing from wearable devices, to genomics. Belle's research indicates that "After decades of being the technological laggard, the field of medicine has begun to acclimatize to today's digital data age. New technologies make it possible to capture vast amounts of information about each individual patient over a large timescale. However, despite the advent of medical electronics, the data captured and gathered from these patients has remained vastly underutilized and thus wasted" (Belle, et al, 2015).

The challenge and potential in Healthcare data lies in the ability to combine traditional forms of data with new forms of data both individually and on a population level," (Raghupathi and Raghupathi, 2014). In addition, Healthcare Big data comes in many forms ranging from electronic health records (EHR), clinical decision support systems, and external sources including government sources, laboratories, pharmacies, insurance companies \& HMO's,etc.), often in multiple formats, (Raghupathi and Raghupathi, 2014). Belle, et al., adds that "Big data analytics which leverages legions of disparate, structured, and unstructured data sources is going to play a vital role in how healthcare is practiced in the future (Belle, et al., 2015). 
The diagram in Figure 1 below displays the input of Big Data sources as raw data which is transformed via various platforms and tools into useful information through Big Data Analytics and applications.

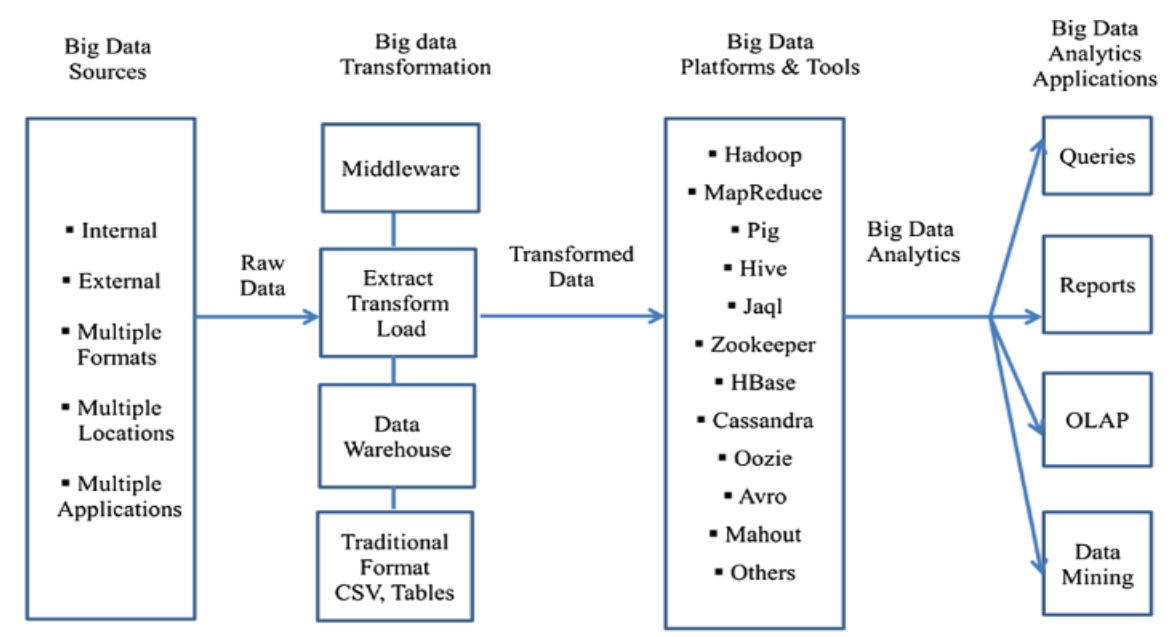

Figure 1. An Applied Conceptual Architecture of Big Data Analytics by Raghupathi and Raghupathi (2014)

Interpreting diagnostic information from medical images can be difficult. "Images are estimated to make up as much as 90 percent of all medical data today, but it can be difficult for physicians to glean important information from them, according to a senior manager for intelligent information systems at IBM Research,” (Orcutt, 2015).

Recently, IBM has announced that it will be using its "Watson" computer to process billions of medical images in order to enable doctors to diagnose diseases more accurately. Watson will use "Deep Learning" which is a form of Artificial Intelligence, to interpret data from many sources to help in medical decisions. "Deep Learning involves training a computer to recognize often complex and abstract patterns by feeding large amounts of data through successive networks of artificial neurons, and refining the way those networks respond to the input," (Knight, 2015). "The biggest barrier to using deep learning in medical diagnostics is that so much of the data necessary for training the systems remains isolated in individual institutions, and government regulations can make it difficult to share that information" (Orcut, 2015).

\section{FINDINGS}

\section{The Intersection of Healthcare Challenges}

All of the above mentioned factors and challenges live within the convergence of Ethics, Security, Personal Health Information, which generates and drives Big Data, as illustrated below in Figure 2. 


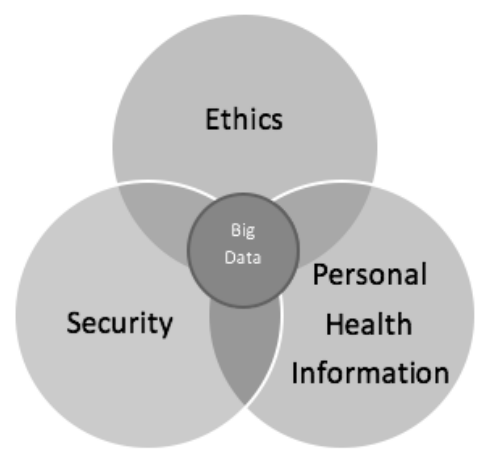

Figure 2. The Interaction of Big Data, Ethics, Security and Healthcare Patient Information

\section{Ethics}

Ethics in healthcare Information Technology is still a relatively new frontier for many. Not simply in the sense of viewing things about a patient that one should not or looking up famous patients but what is happening with the data that systems collect about patients. Of course looking up the records of a famous personality and sharing that information is a HIPAA violation but there are some fine lines and a lot of grey area with respect to ethics. Pharmaceuticals and research companies and willing to and indeed are buying patient data from hospitals and practices. They are purchasing data, usually de-identified, in order to ascertain the number of people afflicted by certain conditions to develop new therapies which can be viewed as a positive thing. Unfortunately, the methods being used and the means of moving that data across systems, could expose Personal Health Information (PHI) to the public. Within the parameters of acquiring this data for the purpose of research or for marketing purposes, patients are often not clearly informed on this practice going on and the notifications to the patient are often buried deep within the Notice of Privacy Practices (NOPP) of many facilities if they exist at all.

In the space of health records, ethical actions by employees at all levels is of paramount importance to the widespread adoption of electronic systems. With so much personal information on patients at the fingertips the lure of potentially unethical or illegal actions is never far away. Companies must put into place training programs and meaningful compliance protocols and reporting mechanisms to keep data safe and protected. Unfortunately, training programs are simply not enough and more rigid administrative safeguards must be put into place.

\section{Security}

Security of data is constantly an issue in healthcare. The security of systems always seems to be a hotly debated topic between those whom call themselves experts, systems administrators and the end users. To some, security means locking systems down so tightly with group policies and specific file-level permissions which is often met with much resistance from the end users who are just trying to do their work. System administrators are working to find the right level of balance between tight security practices and enough user-level access to files that the work of the doctors is not impeded. It is important that we do not get lost in security as a software-only policy. The security of the applications and systems must be coupled closely with a security culture and must be embraced by all users within a company. Too many times do users walk away from their computers, just for a second, to attend to a need and often expose PHI that was left open on their screens to passersby? Arguably, the facet of security that is the most difficult to create and enforce is not with the applications but with the people using them who often do not consider any threats whatsoever let alone anything outside of their specific work area. End users do not want to put in another password nor do they not want to lock their computers, they just want to do their work with as few impediments as possibly without regard as to how it is helping protect health information.

The security layer overlaps both the storage of personal health information and the mechanisms that allow other companies or people to access the information. Security policies must be enacted, monitored and altered over time to make sure that they do not become archaic and potentially flawed. Programs involving system interoperability can raise more security concerns as the automated system-to-system information exchanges typically occur in hear time and are often only monitored after an issue or breech has occurred. Ethics and security deserve more scrutiny 
within the organization. Users should not be accessing the systems with administrator credentials or rights as those accounts should be used primarily when working on the systems and not when doing day-to-day work tasks. When users access the systems in these general accounts the impact of user access applications is diminished and could hide the activities of individuals. A critical aspect of security is the expectation that all users have their own individual accounts that are subject to usage logging and all accessing of common accounts should be avoided when possible.

\section{Personal Health Information and Electronic Health Records (EHR's)}

Patient information is at the heart of the technology. As described in their paper, Ash, et al, focuses on the issue of data input and retrieval of patient information. "Increasingly, the entry and retrieval of information into and from a PCIS are a core activity in health care work," (Ash, et al, 2004). Doctors are spending a larger percentage of their time entering patient data. In an article by Sumathi, a study in JAMA Internal medicine in 2015 revealed that doctors looked at a computer screen a lot during patient visits. The same article discusses a Northwestern University study, in which doctors who use EHR (Electronic Health Records) spend about one-third of patient visits looking at a computer screen, (Sumathi, 2015).

Are EMR and EHR's improving patient care? According to a study conducted by Asan, Smith and Montegue, (2014), physicians spent less time interacting with patients and more time reading EHR's. "Not surprisingly, a significantly smaller proportion of physician time was spent gazing at the patient when using an EHR compared with when using a paper chart," (Asan, Smith and Montegue, 2014).

\section{Big Data and Population Health}

Population Health looks at the patient population for a system to determine missed treatment opportunities for specific patients and seeks to help providers better identify potentially underserved patients. This system can be at the micro level and focus just on the patients of an out clinic facility or can it can be applied at a more macro-level to look at the trends in a geographical area, state or an entire country. As the provider reimbursement landscape changes from the older Fee-For Service model wherein doctors are paid based on treating a patient to the new Payfor-Performance-type systems which adjust payments based on a more holistic view of individual patient's overall health Population Health programs become increasingly important. Doctors are going to be paid not for just seeing a patient but can receive higher payments or incentives for keeping patients out of emergency rooms by proactively reaching out to patients to provide better preventative treatments. For example, doctors can receive high payments from insurers for regimentally seeing their asthmatic patients every three months to monitor and adjust controller medications instead of the patient scheduling an appointment when the meds are starting to fail or the patient having to visit the emergency room. Enabled by the digitization of the patient data into a discrete format instead of handwriting on paper charts, Population Health applications can comb through thousands of records in a second and return lists of patients meeting the desired requirements then assist the practices in outreach thus not only saving time but potentially also saving lives.

For Population Health to be the most effective, data from various faculties a patient might visit must reside together in a single location for the applications to get the best results and a clear overview of the patient's characteristics. It is for this reason that systems must improve the communication between one another, particularly with the Primary Care Providers who are often the ones employing Population Health programs. In many systems, laboratory information is already communicated in real-time to providers but much of the communication between external systems is still taking place via fax. Sharing data via fax is not only a HIPAA breech waiting to happen but it is also non-discrete data and cannot exist as discrete, reportable data within Electronic Health Records systems without labor-intensive manual entry. Some measures in Meaningful Use are seeking to eliminate the fax and assist in the digitization process by incentivizing systems to utilize secure messaging systems with national standards to communicate patient information. This already digitized information can readily be consumed by most EHRs and facilitate a faster response from Population Health mechanisms.

With the ability to "mine" databases looking for treatment opportunities, it is important that these programs be used in the wellness of patients and not filling up schedule books. These systems are designed to help providers keep closer tabs on the health of their patients and to more easily identify trends, not try to make sure the exam rooms are 
full during historically slow times or recalling a patient that may not necessarily be due for treatment. Other ethical concerns exist, such as producing patient lists with certain conditions to be included in lucrative drug trials or selling the information to outside entities for any purpose. With greater and faster capabilities to look at larger amounts of patient data, it is easy to see how some applications of technology can be used to generate additional income but with high ethical decisions, practices and policies of leaders and systems these powerful tools can be used for their intended purposes and help improve the overall health of populations.

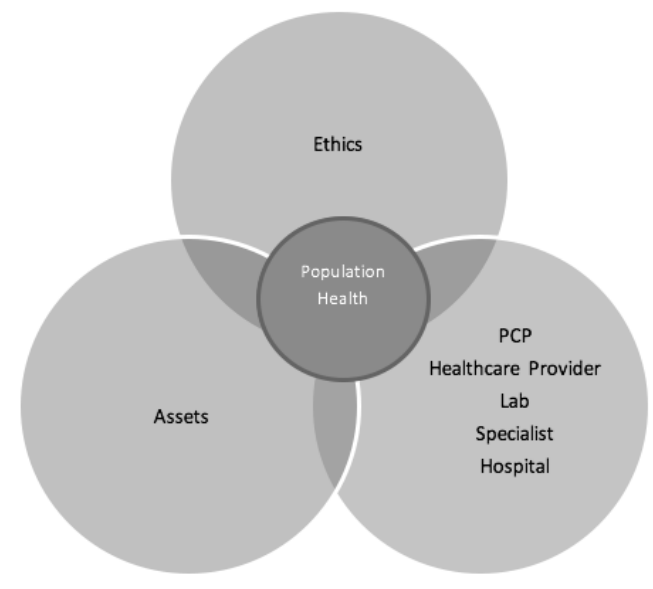

Figure 3. Diagram Displaying the Challenges Around Population Health

In her article "Identifying Care Disparities with Population Health Management," Bresnick describes the gaps and disparities in healthcare as the "Health IT Ecosystem" matures and develops a clearer understanding of patient needs, access to services, and access to self-service. The key to figuring out patient needs and experiences is through quality of data and a better understanding of the data that is gathered, (Bresnick, n.d.).

\section{The Digital Divide in Healthcare}

Is there a Digital Divide in healthcare? Healthcare systems of every size are operating on strict budgets and may not all have the means to deploy expensive Electronic Health Record systems. The applications are incredibly expensive from a software licensing aspect and can often cost close to $\$ 20,000$ for just licensing a doctor which doesn't include the application and ancillary module costs like those needed for Population Health. The infrastructure for some systems that cannot be cloud-based can cause prices to soar and then there is the staffing to maintain the system that must be acquired. In many situations, smaller private practices have opted to not use an electronic system and instead pay the Medicare penalty for not having a system that was imposed by the government. In this scenario, the providers at these facilities do not have the technological capabilities of their electronic counter parts and can find it very difficult to share patient information. Some hospital systems will not share patient information by fax anymore further adding to the difficulties in obtaining patient discharge instructions or emergency room visit notes. Herein lies the great digital divide separating the haves and the have-nots. In these situations it is often the patient who ends up suffering while the providers continue to operate on profits that do not reflect out of pocket expenses for such electronic systems. A patient in the emergency room cannot give permission for a doctor to view records uploaded to an exchange by the PCP because non exist and depending on the situation upon which a patient arrives at an emergency room can have catastrophic consequences. When needed laboratory work, patients are often left to carry paper lab requisitions and may be responsible for obtaining results on their own to transfer back to their physician.

While we have only just started down the unpaved path of widespread Electronic Health Record utilization the situation can only get better. There are surely gaps in the capabilities of systems and divides between those with and without electronic systems but they will close in time. The smoothing of the bumps and gaps will allow everyone to have better access to treatment and give providers a more complete picture of our health. For this to happen, everyone must get on board. The government must step in and create meaningful regulations, software vendors 
need to have time and develop talent to hone their systems and interoperability between systems must be built within a framework that works and can be easily deployed.

\section{CONCLUSION AND FUTURE RESEARCH}

Information Systems challenges will constantly be present as interoperability, efficiency and portability remain an issue in Healthcare. "In sum, no system is fool proof. This points to the increasingly important role technology must play in enforcing HIPAA compliance," (Piliouras, et al, 2015). Healthcare has become the major users of cutting edge technologies where data has become their most important asset and risk. As technologies evolve, especially with the integration of mobile applications, security and the protection of this data becomes even more prevalent. Challenges include maintaining a sustainable security initiative that assures customers that their data is secure. "A trustworthy security program is not an option, but an obligation. In a world where personal data is proliferating at an exponential rate, it must be properly safeguarded lest it fall into the wrong hands," (Robichau, 2014). Other issues hindering effective use of technologies is the actual adoption of these technologies. "Most studies have focused on EHR adoption. Relatively little attention has been paid to the capabilities of those systems and the degree to which physicians with EHR's (Electronic Health Records) actually use them," (Holden \& Karsh, 2009).

This leads into the discussion of future research. Analysis of successes and challenges of Healthcare technologies are ongoing and needs to be investigated. Future research on this endeavor points to the need for further data collection, discussion, interviews, and surveys with administrators, vendors, end users, physicians and Healthcare technology professionals to gather additional data and to provide clarification concerning best practices, compliance, improvements and trends in healthcare technologies.

\section{REFERENCES}

Asan, O., Smith, P., Montague, E. (2014).More screen time, less face time - implications for EHR design. Journal of Evaluation In Clinical Practice, 20(3), 1-6. doi:10.1111/jep.12182

Ash, J. S., Berg, M., \& Coiera, E. (2004). Some unintended consequences of information technology in health care: the nature of patient care information system-related errors. Journal of the American Medical Informatics Association, 11(2), 104-112

Belle, A., Thiagarajan, R., Soroushmehr, S., Reza, M., Navidi, F., Beard, D., Najarian, K. (2015). Big Data Analytics.in Healthcare. BioMed Research International. 7/2/2015, Vol. 2015, p1-16. 16p. DOI: $10.1155 / 2015 / 370194$.

Blumenthal, D., M.D., M.P.P. (2009). Stimulating the Adoption of health Information Technology. New England Journal of Medicine, 2009; 360:1477-1479. April 9, 2009. DOI: 10.1056/NEJMp0901592

Brenner, S. K. , Kaushal, R., Grinspan, Z., Joyce, C., Kim, I., R. J. Allard, R. J., Delgado, D., Abramson, E. L. (2015). Effects of health information technology on patient outcomes: a systematic review. Journal of the American Medical Informatics Association

Bresnick, J. (2015). Healthcare Big Data Analytics Confuses Half of Providers. Health IT Analytics. Accessed May 11, 2016 from http://healthitanalytics.com/news/healthcare-big-data-analytics-confuses-half-ofproviders

Bresnick, J. (n.d). Identifying Care Disparities with Population Health Management. http:/healthitanalytics.com/features/identifying-care-disparities-for-population-health-management 
Dash, M. (2016). Key Technology Challenges for the Healthcare Industry. Healthcare Industry News. April 6, 2016.

Ghosh, R. (2014). Healthcare analytics: A year of many challenges. OR-MS Today, 41(6), 18.

Healthit.gov (2016). Health IT Regulations; Meaningful Use Regulations. Retrieved from Healthit.gov, May 11, 2016: https://www.healthit.gov/policy-researchers-implementers/meaningful-use-regulations

Health Information Privacy "HIPAA." Accessed on May 11, 2016 from the U.S. Department of health and Human Services. http://www.hhs.gov/hipaa/

Holden, R. \& Karsh, B.T. (2009). The Technology Acceptance Model: Its past and its future in health care. Journal of Biomedical Informatics 43 (2010) 159-172

Knight, W. (2015). IBM pushes Deep Learning with a Watson upgrade. MIT Technology Review. July 9, 2015. https://www.technologyreview.com/s/539226/ibm-pushes-deep-learning-with-a-watson-upgrade/

Mostashari, F, Dr. (2012). Meaningful Use Stage 2: A Giant Leap in Data Exchange. https://www.healthit.gov/buzz-blog/meaningful-use/meaningful-use-stage-2/

Orcut, M. (2015). Why IBM Just Bought Billions of Medical Images for Watson to Look At. MIT Technology Review. August 11, 2015. https://www.technologyreview.com/s/540141/why-ibm-just-bought-billions-ofmedical-images-for-watson-to-look-at/

Piliouras, T. C., Suss, R. J., Yu, P. L., Kachalia, S. V., Bangera, R. S., Kalra, R. R., \& Maniyar, M. P. (2015). The rise of mobile technology in healthcare: The challenge of securing teleradiology. Paper presented at the 1-6. doi:10.1109/CEWIT.2015.7338167

Raghupathi, W.and Raghupathi, R. (2014). Health Information Science and Systems, 2014, 2:3. http://www.hissjournal.com/content/2/1/3

Robichau, B. P. (2014;2015;). Healthcare information privacy and security: Regulatory compliance and data security in the age of electronic health records (1st ed.). Berkeley, CA: Apress. doi:10.1007/978-1-4302$6677-8$

Stoltenberg Consulting, Inc. (2015). Third Annual Health IT Industry Outlook Survey. $3^{\text {rd }}$ annual HIMMS conference. Chicago, IL. 2015. Accessed May 11, 2016. http://www.stoltenberg.com/documents/surveys/Third\%20Annual\%20HIT\%20Industry\%20Outlook\%20Su rvey.pdf

Sumathi, R. (2015). Is Your Doctor Getting Too Much Screen Time? The Wall Street Journal. December 14, 2015. http://www.wsj.com/articles/is-your-doctor-getting-too-much-screen-time-1450118616

Tan, S. S., Gao, G., \& Koch, S. (2015). Big data and analytics in healthcare. Methods of Information in Medicine, $54(6), 546$. 